\title{
Germanica
}

\section{Deux lettres de Jamaïque sur la Révolution}

Anna Seghers : La Lumière sur le gibet, Heiner Müller : La Mission

Zwei Briefe aus Jamaika über die Revolution

\section{Isabelle Vodoz}

\section{(2) OpenEdition}

\section{Journals}

Édition électronique

URL : http://journals.openedition.org/germanica/2536

DOI : 10.4000/germanica. 2536

ISSN : 2107-0784

Éditeur

Université de Lille

Édition imprimée

Date de publication : 31 décembre 1989

Pagination : 181-194

ISSN : 0984-2632

Référence électronique

Isabelle Vodoz, « Deux lettres de Jamaïque sur la Révolution », Germanica [En ligne], 6| 1989, mis en ligne le 04 décembre 2014, consulté le 06 octobre 2020. URL : http://journals.openedition.org/ germanica/2536 ; DOI : https://doi.org/10.4000/germanica.2536

Ce document a été généré automatiquement le 6 octobre 2020.

(c) Tous droits réservés 


\title{
Deux lettres de Jamaïque sur la Révolution
}

\author{
Anna Seghers : La Lumière sur le gibet, Heiner Müller : La Mission \\ Zwei Briefe aus Jamaika über die Revolution
}

Isabelle Vodoz

\section{NOTE DE L'AUTEUR}

La nouvelle d'Anna Seghers fait partie du volume XI de ses œuvres parues aux éditions Aufbau-Verlag.

Les œuvres de Heiner Müller sont citées d'après l'édition complète en allemand, dont 9 volumes sont parus jusqu'ici au Rotbuch Verlag Berlin, à l'exception de La Mission que je cite dans la traduction française.

Les deux interviews de Heiner Müller sont parues, l'une dans le n 1733 de France Nouvelle (29/1/79), tandis que je cite l'interview extraite du Spiegel (19/1983) dans sa traduction française, publiée dans le ${ }^{\circ} 56$ de la revue théâtre / public.

1 «Avez-vous jamais vous-même inventé un sujet dramatique? » À cette question posée par un journaliste du Spiegel Heiner Müller répond: «Je ne crois pas, non». Mais il ajoute: "Chaque texte nouveau est en relation avec quantité de textes antérieurs d'autres auteurs ; il modifie aussi le regard qu'on pose sur eux. Mon commerce avec des sujets et des textes anciens est aussi un commerce avec un "après". C'est, si vous voulez, un dialogue avec les morts ».

Double dialogue avec les morts que La Mission, pièce écrite en 1979 et qui porte comme sous-titre "Souvenirs d'une révolution", avec la précision suivante: "Cette pièce reprend des thèmes de la nouvelle de Anna Seghers La Lumière sur le gibet ». Dialogue donc avec des morts de la Révolution française, dialogue aussi avec la vision de la révolution qui était en 1961 celle de la grande romancière. 
Je n'ai pas eu recours à la traduction française de Das Licht auf dem Galgen (éditions de l'Arche), tandis que mes citations de Der Auftrag sont empruntées à la traduction de J. Jourdheuil et H. Schwarzinger, parue en 1982 aux éditions de Minuit. Je proposerais cependant deux modifications : "Remémoration d'une révolution » me semble mieux prendre en compte le sens actif convoyé par "Erinnerung an eine Revolution»; d'autre part, à la place de "reprendre des thèmes ", je suggérerais "emprunter des motifs ", qui rend plus exactement justice à la démarche de Heiner Müller. Le rapprochement des deux textes révèle en effet que les motifs empruntés sont de tous ordres, allant de la fable dans son ensemble jusqu'à des points de détail, en passant par les personnages ou des précisions historiques ou géographiques dont, à son habitude, il fait un usage très libre. La pièce n'est donc en rien une adaptation de la nouvelle, pourtant, malgré ce décalage, la mise en parallèle des deux œuvres me permettra d'utiliser La Lumière sur le gibet pour éclairer La Mission.

4 La longue nouvelle (environ 120 pages) d'Anna Seghers reprend le schéma classique de la «Rahmenerzählung». La place du narrateur est tenue par un marin, Malbec, de passage à Paris entre deux voyages, et qu'un de ses amis, mort depuis, a chargé de remettre une lettre à un dénommé Antoine. Le messager intervient parfois dans le cours de l'histoire pour commenter certains épisodes et c'est lui qui, à la fin de la nouvelle, tirera la leçon des événements. Voici la lettre qu'il apporte :

J'utilise cette occasion pour vous informer que les citoyens Debuisson et Sasportas ont été arrêtés. Sasportas est passé en jugement sur-le-champ et a été exécuté à Kingston. Debuisson a quitté la Jamaïque sur un vaisseau anglais. Il s'en est tiré en avouant.

5 La pièce de Heiner Müller ne comprend qu'une trentaine de pages en allemand et calque son mouvement général sur celui de la nouvelle, à laquelle sont empruntés et le lieu de l'action et les personnages principaux. Ici, plus de " Rahmenerzählung », Heiner Müller travaille en "flash-back » et les seules réflexions sur les faits qui se déroulent sous nos yeux sont ceux des protagonistes de l'action. La pièce s'ouvre sur la lettre, sans mention explicite de celui qui la lit, et le metteur en scène a donc entière latitude d'en confier la lecture à tel ou tel personnage ou à une voix-off. Simple constat chez Anna Seghers, elle concentre la quasi-totalité des thèmes abordés dans La Mission et c'est pourquoi sa lecture commentée me servira de fil directeur.

Galloudec à Antoine. J'écris cette lettre sur mon lit de mort. J'écris en mon nom et au nom du citoyen Sasportas qui a été pendu à Port-Royal. Je vous informe que nous devons nous démettre de la mission que la Convention nous avait confiée par votre intermédiaire, puisque nous n'avons pu la remplir. Peut-être d'autres feront-ils mieux. Vous n'aurez plus de nouvelles de Debuisson, il va bien. À croire que les traîtres sont prospères quand les peuples pataugent dans le sang. Le monde est ainsi fait et ce n'est pas bien ainsi. Excusez mon écriture, ils m'ont amputé d'une jambe et j'écris dans la fièvre. J'espère que cette lettre vous trouvera en bonne santé et je vous prie d'agréer l'expression de mon salut républicain.

\section{Galloudec à Antoine}

6 Parmi la multitude des personnages d'Anna Seghers quatre seulement conservent chez Heiner Müller leur statut de personnage, c'est-à-dire en particulier leur nom propre. Malbec, le messager, est réduit au rôle succinct d'un « matelot » anonyme, cependant que la femme d'Antoine n'a plus droit qu'à quelques répliques. Antoine par contre, malgré la brièveté de son apparition, sert à Heiner Müller à esquisser l'image d'une 
France où la Révolution a fait place au Consulat, puis à l'Empire. Cet ancien conventionnel, mélange de jactance - il évoque complaisamment les temps glorieux où tombaient les têtes des aristocrates ou ses combats en Vendée - et de pusillanimité craignant que son passé jacobin ne l'ait rendu suspect il hésite à se reconnaître comme le destinataire de la lettre - fonctionne comme le porte-parole amer et désabusé des déçus de la révolution.

Galloudec, l'auteur de la lettre, est chez Anna Seghers un marin dont on sait seulement que ses voyages l'ont rendu le témoin admiratif du soulèvement des esclaves en Haïti sous la conduite de Toussaint Louverture. Heiner Müller le caractérise comme « un paysan de Bretagne », ce qui revient à l'inscrire plus nettement dans le tissu français : représentant en quelque sorte du peuple de France, il est aussi le blanc qui s'identifie à la cause de la Révolution aux Caraïbes. Dans le dernier entretien avec ses deux camarades il choisit nettement son camp : «Je viens avec toi, Sasportas. Nous devons tous mourir un jour, Debuisson. Et c'est tout ce que nous avons encore en commun. Après le massacre à la Guadeloupe, au milieu d'un monceau de cadavres, tous noirs, ils en ont trouvé un blanc, qui était tout aussi mort. Toi en tout cas, ça ne peut plus t'arriver, Debuisson. Tu es dehors ».

J'écris cette lettre sur mon lit de mort. J'écris en mon nom et au nom du citoyen Sasportas qui a été pendu à Port-Royal.

8 La différence de conception entre les deux Sasportas, celui de la pièce et celui de la nouvelle, est à mon sens en étroite liaison avec le contexte historique où sont nées les deux œuvres. Dans la nouvelle Sasportas est un jeune Juif d'origine espagnole qui possède quelques connaissances médicales et qui, à ce titre, et par admiration pour Debuisson plutôt que par conviction révolutionnaire, accompagne celui-ci pour l'aider dans sa mission. Au contact de la société esclavagiste de Jamaïque s'opère en lui une sorte de "prise de conscience", il s'engage de plus en plus activement aux côtés des opprimés avec lesquels il noue des liens, en particulier à travers l'amour et l'aide que lui apporte une jeune esclave noire.

9 Les hasards de l'émigration ont conduit Anna Seghers au Mexique, d'où elle reviendra en 1947. Peut-être est-ce cette expérience mexicaine qui l'a amenée, autour et à partir des années 50, à choisir le continent américain, spécialement les Caraïbes, comme cadre de plusieurs de ses nouvelles. C'est en tout cas dans l'esprit de l'internationalisme prolétarien qu'elle y aborde les problèmes de l'esclavage et des guerres de libération des esclaves. La Lumière sur le gibet constitue le troisième panneau d'un triptyque, dont les deux premiers volets datent de 1949 et ont respectivement pour titre Le Mariage en Haïti et Réintroduction de l'esclavage en Guadeloupe. En 1980, trois ans avant sa mort, la romancière publie une nouvelle trilogie « exotique » intitulée Trois femmes de Haïti.

Tout autre est la perspective de Heiner Müller. Il est en effet d'un temps qui s'est trouvé confronté tant aux échecs qu'aux suites désastreuses des victoires des guerres de libération nationale, cependant qu'en Allemagne, comme dans les autres pays européens, se pose de manière de plus en plus aiguë la question du rapport aux travailleurs immigrés. Son désenchantement s'accompagne ainsi de l'idée qu'il n'y a plus grand chose à attendre de ses concitoyens allemands, surtout de ceux de RFA, à propos desquels il déclare, dans l'interview au Spiegel déjà citée : «Je crois qu'en RFA, à l'heure actuelle, seuls les travailleurs immigrés seraient aptes à écrire de véritables pièces. Les autres ne sont capables que de photographier le public. Ils sont le public. Ce 
qui est constitutif d'un drame, au fond, ne peut être vécu et articulé que par des travailleurs immigrés ».

11 Rien d'étonnant donc à ce que Sasportas, présenté d'entrée de jeu comme le « citoyen » Sasportas, soit devenu dans la pièce un ancien esclave noir, lequel a pris en charge sa propre libération avec un radicalisme qui le conduit parfois à projeter la question raciale comme telle au premier plan. Les affrontements avec Debuisson sont assurément les plus violents : «Le théâtre de la révolution blanche est terminé. Nous te condamnons à mort, Victor Debuisson. Parce que ta peau est blanche. Parce que tes pensées sont blanches sous ta peau blanche ». Mais Galloudec non plus n'est pas épargné. Quand il s'insurge au nom de leur idéal commun : "Nous ne sommes pas ici pour nous reprocher l'un à l'autre la couleur de notre peau, citoyen Sasportas ", il s'entend répondre durement : « Nous ne serons pas égaux tant que nous ne nous serons pas enlevé la peau l'un à l'autre». Et ce radicalisme, qui confère une incontestable grandeur au personnage - ce n'est pas un hasard si dans une scène un peu grand guignolesque comme il les aime, et intercalée dans l'action, Heiner Müller prête à Sasportas le masque et le discours de Robespierre - lui fait en même temps perdre le caractère d'exemplarité qu'il avait encore dans la nouvelle. Révolutionnaire à part entière, Sasportas ne sortira, si l'on peut oser ce jeu de mots, pas plus blanc que les autres des crimes de la Révolution, car personne ne saurait se laver les mains du sang qu'elle fait couler.

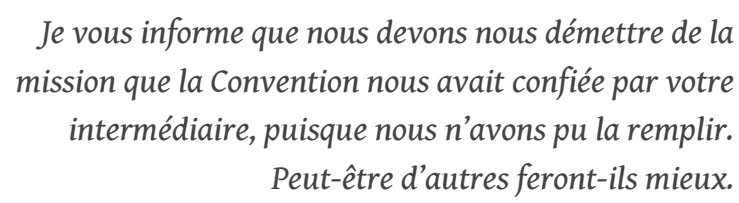

12 La comparaison entre les deux lettres est ici très instructive. Le Galloudec d'Anna Seghers se borne à apporter des informations sur le sort réservé à ses deux camarades. Dans la pièce, par contre, c'est la mission non remplie qui tourmente le mourant, animé qu'il est de la conviction que cet échec n'est pas dû à un concours de circonstances malheureux mais au fait que les trois émissaires ont failli à leur tâche. Je reviendrai ultérieurement sur le caractère central de cette mission qui donne son titre à la pièce, me contentant pour l'instant d'en donner lecture, telle qu'elle est énoncée dans la pièce, par la voix d'un énonciateur non déterminé :

Nous étions arrivés à la Jamaïque, trois émissaires de la Convention, nos noms Debuisson, Galloudec, Sasportas, notre mission le soulèvement des esclaves contre le règne de la couronne d'Angleterre au nom de la république de France. Qui est la mère patrie de la révolution, la terreur des trônes, l'espoir des pauvres. Où tous les hommes sont égaux sous le couperet de la justice. Qui manque de pain pour la famine de ses faubourgs, mais non de mains pour porter dans tous les pays la torche de la liberté égalité fraternité.

Vous n'aurez pas de nouvelles de Debuisson, il va bien.

À croire que les traîtres sont prospères quand les peuples pataugent dans le sang. Le monde est ainsi fait et ce n'est pas bien ainsi.

La ligne de démarcation entre les deux œuvres passe également par le rôle attribué au personnage de Debuisson, et ce, dès la lettre initiale. Dans un cas comme dans l'autre il s'agit du descendant d'une famille de propriétaires esclavagistes qui a embrassé avec enthousiasme la cause de la révolution, à telle enseigne qu'il a été chargé par la Convention de revenir dans son île pour aider au soulèvement des esclaves. Adoptant 
pour ainsi dire le point de vue marxiste classique, Anna Seghers observe avec une sorte d'impartialité sociologique comment, replongé dans son milieu d'origine, il retrouve ses vieux réflexes de classe, ce qui, ajouté aux difficultés de la tâche, finit par émousser sa combativité et entamer sa croyance en la justesse de la cause pour laquelle il a entrepris de lutter. L'épreuve finale le trouve sans résistance et il passe aux aveux pour garder la vie sauve.

14 La tension est autrement plus grande chez Heiner Müller à qui le personnage de Debuisson permet d'introduire un thème, récurrent dans son œuvre, celui de la trahison. Des raisons biographiques expliquent en partie l'importance que la trahison occupe dans son théâtre. Dans une nouvelle de 1958, Le Père, Heiner Müller raconte en effet comment l'enfant de quatre ans qu'il était en 1933 fit semblant de dormir pour ne pas avoir à dire au revoir à son père, emmené par la Gestapo, et comment, quelques années plus tard, ce même père, libéré mais au chômage, l'a incité à écrire une rédaction où l'enfant déclarait être content que Hitler construisît des autoroutes. L'horreur de la trahison c'est aussi, comme le dit Galloudec, que les traîtres soient prospères, et la grand-mère du petit garçon n'a pas manqué de lui expliquer que s'il devait se contenter de margarine, alors qu'elle-même mangeait du beurre, c'était bien parce que son père était contre Hitler. Il y a donc une volupté de la trahison à laquelle Debuisson supplie ses camarades de l'aider à résister: "J'ai peur de la beauté du monde, Galloudec. Je sais bien qu'elle est le masque de la trahison. [...] Tuez-moi avant que je vous trahisse. Je redoute, Sasportas, la honte d'être heureux en ce monde ». Mais ceux-ci se détournent de lui, Sasportas avec haine, Galloudec avec mépris. Debuisson, resté seul en scène, lutte en vain contre la trahison - représentée par Heiner Müller sous les traits d'une femme lubrique - et la pièce se clôt sur sa défaite.

Malgré cette insistance du motif je pense que ce serait une erreur de faire de la trahison le thème central de la pièce et que la question axiale, celle, en fin de compte, autour de laquelle se rejoignent Heiner Müller et Anna Seghers - tous deux auteurs de RDA, mais qu'une génération sépare - demeure le problème de leur rapport à la révolution.

Dans La Lumière sur le gibet Anna Seghers fait œuvre de mémoire : il importe avant tout de sauver de l'oubli les vaincus de l'histoire afin que leur héroïsme serve d'exemple à ceux pour qui la lutte continue et qui seront peut-être les vainqueurs de demain. Une révolution n'est jamais pour elle qu'un jalon sur la longue route qui mène les damnés de la terre à la révolution. En ce sens, la Révolution française et ses prolongements aux Caraïbes ne représentent que des péripéties, douloureuses mais exaltantes. Le thème du souvenir sous-tend toute la nouvelle. Si Antoine finit par accepter la lettre c'est qu'il prend soudain conscience que "ce petit morceau de papier» est un "véritable témoignage ", le seul qui reste de ces obscurs combattants. Galloudec, pour sa part, demeure obsédé dans son délire par l'action et la mort de Sasportas, que symbolise « la lumière jaillie du haut de la potence [qui] brillait en sa direction». Et la voix du messager Malbec s'élève enfin pour rassembler en faisceau le souvenir des anonymes :

Maintenant je sais ce qu'il voulait dire avec sa lumière. Elle n'éclaire pas seulement vers l'arrière la vie de Sasportas, elle éclaire tous ceux à qui Sasportas a eu affaire ; autrement ils auraient disparu sans laisser de trace dans des eaux profondes ou dans la forêt vierge; leurs noms ne sont inscrits dans aucun livre et sur aucun monument; peut-être n'avaient-ils pas même de vrais noms. Bedford, Cuffee étaient-ce là de vrais noms? Et pourtant ils ont accompli des choses vraies, des choses importantes, aussi vraies et aussi importantes que celles qui ont été accomplies ici à Paris. 
17 Pour les personnages de Heiner Müller, en revanche, la lumière jaillie de la potence semble s'être éteinte dans le sang où pataugent les peuples. Sans doute est-ce le désir d'excuser son propre renoncement qui pousse Antoine à déclarer : « La liberté conduit le peuple sur les barricades, et quand les morts se réveillent elle porte un uniforme ». Sans doute Debuisson s'efforce-t-il de se convaincre et de convaincre ses camarades du caractère absurde voire criminel de la mission qu'il s'apprête à trahir : "La révolution fatigue, Galloudec. Dans le sommeil des peuples se lèvent les généraux et ils brisent le joug de la liberté si lourd à porter. [...] La révolution n'a plus de patrie, [...] l'esclavage a de multiples visages, nous n'avons pas encore vu le dernier, ni toi Sasportas, ni nous Galloudec, ce que nous avons pris pour l'aurore de la liberté n'était peut-être qu'un nouvel esclavage plus effroyable... ». Il n'en reste pas moins qu'un énoncé scande la pièce, dont les capitales viennent souligner l'importance : LA RÉVOLUTION EST LE MASQUE DE LA MORT. LA MORT EST LE MASQUE DE LA RÉVOLUTION.

Est-ce à dire que Heiner Müller doit désormais être rangé aux côtés des transfuges échaudés de la révolution, de ceux dont la seule perspective est devenue la plate philosophie des droits de l'homme? Les choses ne sont pas si simples. Jouissant d'une solide notoriété en RFA, où il travaille fréquemment, et malgré les difficultés - aussitôt exploitées par les médias de l'Ouest - qu'il a pu avoir avec la censure dans son pays, Heiner Müller se considère comme un écrivain de RDA, et il souligne volontiers, de manière parfois légèrement provocatrice, ce qui le sépare de l'autre Allemagne. A propos d'une mise en scène de La Mission il en arrive ainsi, dans le Spiegel, à vitupérer le caractère "voyeur » du public de RFA, qu'il explique par le fait « que ce public-là n'a aucune expérience de la révolution sinon celle tirée du manuel scolaire et de l'idée commune que la révolution est quelque chose de terrible ».

19 Mais dans le même temps, au cours d'une interview à l'hebdomadaire communiste France nouvelle, il indique que le thème central de toutes ses œuvres serait « le décalage entre le temps du sujet et le temps de l'histoire ", décalage qui n'aurait fait que s'accentuer dans ses dernières œuvres, et ajoute : «La naissance du désespoir c'est quand l'écart atteint une telle proportion qu'il détruit l'identité du sujet ». Or cet écart est particulièrement manifeste quand il s'agit d'aborder le problème de la révolution.

Il est révélateur à cet égard de comparer La Mission avec Mauser, pièce didactique qui date de 1970. Mauser raconte l'histoire d'un jeune révolutionnaire qui, confronté à la mission de tuer trois paysans au nom de la révolution, a reculé, et qui, pour cette faute, se voit à son tour condamné à mort. La pièce est consacrée à la discussion entre le chœur et le jeune camarade, qui finit par aller au-devant de la mort « Sachant : le pain quotidien de la révolution / Est la mort de ses ennemis, sachant: l'herbe même / Il nous faut l'arracher afin qu'elle reste verte ». L'idée directrice est que la révolution n'a pas à connaître de l'homme tant qu'elle n'est pas accomplie et que la tâche, pour sanglante qu'elle soit, doit être menée à bien, ce que Heiner Müller résume dans une note sous la forme : «Pour que quelque chose advienne, il faut que quelque chose parte, la première figure de l'espoir est la peur, la première apparition du nouveau l'effroi ». Dans La Mission la perspective a changé, ainsi qu'en témoigne un long monologue qui se situe au centre de la pièce, encadré par deux scènes où s'affrontent les points de vue des trois émissaires. La première de ces scènes les montre, tout juste arrivés à la Jamaïque, se préparant à leur mission, avec en arrière-plan la cage où agonise un esclave noir, puni pour avoir tenté de s'enfuir. Galloudec est, des trois, celui qui s'inquiète du sang qu'ils vont faire couler, inévitablement : "Toujours c'est un seul qui 
meurt. Et on compte les morts ». Sasportas et Debuisson, malgré leur antagonisme sous-jacent, sont, eux, du côté du radicalisme. Au radicalisme concentré et presque haineux de Sasportas : "Quand je partirai d'ici, d'autres seront dans les cages, à peau blanche jusqu'à ce que le soleil la noircisse. Beaucoup alors s'en trouveront mieux. ", s'oppose le radicalisme à la fois désinvolte et résigné, sorte de politique du pire, de Debuisson, qui laisse en fait prévoir sa trahison: "Observe la chair qui meurt dans cette cage. [...] Son gémissement est la Marseillaise des corps sur lesquels se bâtit le nouveau monde. Apprenez cette musique. Nous l'entendrons encore longtemps, de gré ou de force, c'est la musique de la révolution, de notre tâche. [...] Voilà ce que notre tâche accomplit pour nos semblables, peut-être seulement cela ». Dans la seconde scène les places se distribuent différemment. La mission a échoué et l'on a d'un côté Debuisson, qui s'emploie à justifier sa trahison imminente en arguant entre autres du changement de gouvernement à Paris, et de l'autre Sasportas et Galloudec. Ce dernier refuse d'accepter que la justesse d'une cause soit tributaire de considérations de basse politique, tandis que Sasportas, qui voit déjà se dresser devant lui l'image de la potence, s'écrie : « Les morts combattront quand les vivants ne pourront plus. Chaque battement de cœur de la révolution fera de nouveau croître de la chair sur leurs os, du sang dans leurs veines, de la vie dans leur mort. Le soulèvement des morts sera la guerre des paysages, nos armes les forêts, les montagnes, les mers, les déserts du monde. Je serai forêt, montagne, mer, désert. Moi, c'est l'Afrique. Moi, c'est l'Asie. Les deux Amériques c'est moi. Mais cette exaltation, née du besoin qu'éprouve l'individu, à l'approche de la mort, de se persuader que sa vie avait un sens, est probablement la seule lumière sur $L a$ Mission qui pourrait rappeler Anna Seghers.

Au centre de la pièce donc, ce monologue dont, en l'absence d'indication contraire, on peut supposer qu'il est prononcé par Sasportas. Il s'agit d'un récit, d'un rêve peut-être, non situé dans le temps et dans l'espace, et dont la lecture me fait irrésistiblement penser à la nouvelle de Kafka Un message impérial. Un homme, dans un ascenseur, tente de se rendre à une convocation, mais l'ascenseur n'en finit pas de n'arriver nulle part et l'homme «s'abandonne à des spéculations échevelées » :

Je suppose qu'il s'agit d'une mission qui doit m'être confiée. [...] Le chef (qu'en pensée j'appelle Numéro Un) m'attend avec ma mission, moi le défaillant. Peut-être ma mission [...] est-elle devenue caduque du fait de ma négligence, sans objet dans la langue des administrations que j'ai si bien apprise (science superflue !), a classer au dossier que personne ne consultera plus parce qu'elle concernait précisément les dernières mesures possibles pour prévenir la fin du monde dont je vis actuellement le commencement prisonnier de cet ascenseur devenu fou avec mon braceletmontre devenu fou. [...] J'imagine le désespoir de Numéro Un. Son suicide. [...] Je quitte l'ascenseur à l'arrêt suivant et me retrouve sans mission [...] dans la rue d'un village au Pérou. [...] En quoi consiste mon crime. Le monde n'a pas sombré, si toutefois ceci n'est pas un autre monde. Comment accomplir une mission inconnue. Quelle pourrait être ma mission dans cette contrée désolée au-delà de la civilisation. [...] Sur un talus de chemin de fer recouvert d'herbe deux gamins bricolent un bâtard de machine à vapeur et de locomotive, qui se trouve là sur un tronçon de voie ferrée. En européen, je vois au premier regard que c'est peine perdue : ce véhicule ne roulera pas mais je ne le dis pas aux enfants, le travail c'est l'espoir, et je poursuis ma route dans le paysage qui n'a d'autre tâche que d'attendre la disparition de l'homme. qu'avec La Mission, cette « remémoration d'une révolution », Heiner Müller prend, sans 
doute partiellement à l'aveugle, congé de la révolution, et donc de toute mission univoquement représentable.

Dans les œuvres qui suivent, la présence du sang, de la décomposition, de la mort, ne fait que s'accentuer avec, en toile de fond, la menace de la ruine de l'humanité tout entière. À noter également une fascination pour shakespeare, qui intervient durablement dans sa production à travers traductions, mises en scènes et comme matériau de ses propres pièces, ainsi Hamlet-machine (1977), ou encore Anatomie de Titus, chute de Rome, un commentaire de Shakespeare (1984), dont l'exergue : "Ouvrir les veines de l'humanité et comme un livre feuilleter le fleuve de sang", est éclairant sur la conception qu'il en a. Dans Shakespeare en effet la nature, et non le "marécage sanglant des idéologies », est encore le lieu de l'histoire : "Les morts ont leur place dans son théâtre, la nature a le droit de vote ».

Mais je dirais que Heiner Müller est à la fois trop intelligent et trop exigeant sur sa responsabilité d'artiste pour s'abandonner à cette forme du nihilisme contemporain, auquel il préfère, comme il le dit dans son Adieu à la pièce didactique, un «défaitisme constructif ». Dans une interview à France Nouvelle, antérieure à La Mission, il affirmait déjà que, pour un intellectuel, le pire était la résignation, le désespoir étant une « forme active " susceptible de se traduire "par la découverte de quelque chose »; il ne paraît pas avoir varié sur ce point. Si l'artiste n'a pas de "mission », il n'en a pas moins une " tâche ", celle qu'il évoque précisément dans un texte de 1988, intitulé Shakespeare une différence, et dont j'extrais ces lignes : «Le corps-à-corps de la révolution et de la contre-révolution comme figure de base des catastrophes géantes du siècle. Shakespeare est un miroir à travers les temps, notre espoir, un monde qu'il ne réfléchisse plus. [...] L'effroyable, dans ce que reflète Shakespeare, c'est le retour du même. [...] Notre tâche, sinon le reste deviendra statistique et affaire d'ordinateur, c'est le travail sur la différence. Hamlet, celui qui se dérobe, ne l'a pas accompli, tel est son crime ». Le travail productif à accomplir sur la différence c'est aussi celui que pointent ces notes sur son Philoctète : "L'action est modèle, et non pas fable. Elle sert à montrer des attitudes, et non des significations. [...] Ce n'est qu'en modifiant le modèle qu'on peut espérer apprendre de l'histoire ». Et, à titre de dernière illustration de ce que, malgré les apparences, Heiner Müller ne s'est pas laissé complètement envahir par le catastrophisme ambiant, voici la fin du discours que, à l'occasion de la remise du prix Büchner, il a prononcé en 1985 et dédié à Nelson Mandela.

Texte d'un auteur de vingt-trois ans - que les Parques, en lui tranchant les paupières, ont condamné à regarder en face ce monde dont Galloudec écrivait : "Le monde est ainsi fait et ce n'est pas bien ainsi »- le Woyzeck de Büchner se voit convoqué par Heiner Müller sous les traits de l'ange toujours en éveil qui interdit à l'auteur dramatique l'accès du paradis, où s'abritait jusqu'alors l'innocence de l'écriture. Puis il assigne à comparaître Woyzeck lui-même, lointain écho, me semble-t-il, de Sasportas : "Aujourd'hui encore en Afrique il poursuit son chemin de croix à travers l'histoire, le temps ne travaille plus pour lui... ». Et il conclut :

Woyzeck est la blessure béante. Woyzeck vit là où le chien est enterré, le chien s'appelle Woyzeck. C'est sa résurrection que nous attendons dans la crainte et/ou l'espoir, que le chien revienne comme loup. Le loup vient du Sud. Quand le soleil est à son zénith il fait un avec notre ombre, commence, à l'heure de l'incandescence, l'histoire. Et c'est seulement quand l'histoire aura eu lieu que vaudra l'anéantissement commun dans le gel de l'entropie - ou sous sa forme politique 
abrégée, dans l'éclair de l'atome -, qui marquera la fin des utopies et le début d'une réalité au-delà de l'homme.

\section{RÉSUMÉS}

La nouvelle d'Anna Seghers, La Lumière sur le gibet, traite un épisode de la révolution française et a fourni à Heiner Müller la thématique de sa pièce La Mission. Une comparaison entre les deux œuvres permettra de caractériser les différences entre les positions respectives de ces deux auteurs de RDA face à la Révolution française, position qui s'explique en grande partie par leur rapport à la politique. À partir des dernières œuvres de Heiner Müller on tentera ensuite de préciser la pensée politique - qui n'a cessé de se complexifier - de cet auteur, l'une des plus fascinantes personnalités du théâtre d'aujourd'hui.

Anna Seghers' Novelle Das Licht auf dem Galgen behandelt eine Episode aus der Zeit der französischen Revolution und hat Heiner Müller den Stoff zu seinem Theaterstück Der Auftrag geliefert. Ein Vergleich zwischen den zwei Werken wird es ermöglichen, die unterschiedliche Stellungnahme dieser beiden DDR-Autoren der französischen Revolution gegenüber zu charakterisieren, eine Stellungnahme, die sich zum größten Teil aus ihrer eigenen politischen Einstellung erklären läßt. Anhand der letzten Werke Heiner Müllers wird dann der Versuch unternommen, die immer komplexer werdende politische Gedankenwelt dieses Autors, eines der größten und faszinierendsten Dramatiker unserer Zeit, eingehender zu bezeichnen.

\section{AUTEUR}

\section{ISABELLE VODOZ}

Université de Lille III 